\title{
Selection of mother wavelets thresholding methods in denoising multi-channel EEG signals during working memory task
}

\begin{abstract}
The aim of this pilot study was to select the most similar mother wavelet function and the most efficient threshold in order to use with wavelet basis function for the human brain electrical activity during working memory task. A 60 seconds was recorded from the scalp using the Electroencephalography (EEG). 19 electrodes were placed over different sites on the scalp where analyzed for one control subject and one post-stroke patients in the first week of his stroke onset. In this study, forty-five mother wavelet basis functions from orthogonal families with four thresholding methods were used. The selection of mother wavelet functions like Daubechies (db), symlet (sym) and coiflet (coif) and the thresholding methods these are sqtwolog, rigrsure, heursure and minimax are to check mother wavelet functions similarity with the recorded EEG signals during working memory task. The test have been done using four evaluating criteria, namely signal to noise ratio (SNR), peak signal to noise ratio (PSNR) mean square error (MSE) and crosscorelation method (xcorr). Symlet mother wavelet of order 9 (sym9) is the most compatible for all the 19 channels for both EEG datasets that selected to be examined and the best results have been obtained by using the rigrsure thresholding method.
\end{abstract}

Keyword: Mother wavelet; Thresholding methods; Working memory; EEG 